\title{
25 Research Square \\ Correlation of periodontal diseases with intracranial aneurysm formation: novel predictive indicators
}

\section{Keyun Liu}

Beijing Neurosurgical Institute and Beijing Tiantan Hospital, Capital Medical University

Jia Sun

Tianjin Stomatological Hospital, Hospital of Stomatology, Nankai University

\section{Lingling Shao}

Beijing Tuberculosis and Thoracic Tumor Institute, Beijing Chest Hospital, Capital Medical University

Hongwei He

Beijing Neurosurgical Institute and Beijing Tiantan Hospital, Capital Medical University

Qinglin Liu

Beijing Neurosurgical Institute and Beijing Tiantan Hospital, Capital Medical University

\section{Youxiang Li}

Beijing Neurosurgical Institute and Beijing Tiantan Hospital, Capital Medical University

Huijian Ge (D 474025129@qq.com )

Beijing Neurosurgical Institute and Beijing Tiantan Hospital, Capital Medical University

\section{Research}

Keywords: Gingivitis, Intracranial aneurysms, Inflammation, Periodontitis

Posted Date: February 1st, 2021

DOl: https://doi.org/10.21203/rs.3.rs-36803/v2

License: (c) (1) This work is licensed under a Creative Commons Attribution 4.0 International License.

Read Full License 


\section{Abstract}

Background and purpose: We investigated whether periodontal diseases, specifically, periodontitis and gingivitis, could be risk factors of the incidence of intracranial aneurysms (IAs).

Methods: We performed a case-control study to compare the differences in the periodontal disease parameters of 281 cases that were divided into the IAs group and non-IAs group. All cases underwent complete radiographic examination for IAs and examination for periodontal health.

Results: Comparing with those in the non-IAs group, the cases in the IAs group were older ( $53.95 \pm 8.56$ vs $47.79 \pm 12.33, p<0.001)$ and had a higher incidence of hypertension (76 vs $34, p=0.006)$. Univariate logistic regression analysis revealed that age ( $>50$ years) and hypertension were predictive risk factors of aneurysm formation (odds ratio [OR] 1.047, 95\% confidence interval [95\% Cl] 1.022-1.073, $p<0.001$ and OR $2.047,95 \% \mathrm{Cl} 1.232-3.401, \mathrm{p}=0.006)$. In addition, univariate and multivariate logistic regression analyses showed that the parameters of periodontal diseases, including gingival index, plaque index, clinical attachment loss, and alveolar bone loss, were significantly associated with the occurrence of IAs (all $p<0.05$ ). For further statistical investigation, the parameters of periodontal diseases were divided into four layers based on the quartered data. Poorer periodontal health condition (especially gingival

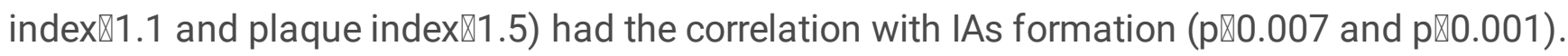

Conclusion: Severe gingivitis or periodontitis combining with hypertension, are significantly associated with the incidence of IAs.

\section{Introduction}

Intracranial aneurysms (IAs) are pathological dilatations of cerebral arteries; they are most often saccular in shape and frequently found in proximal cerebral artery bifurcations ${ }^{[1]}$. Subarachnoid hemorrhage caused by IA rupture affects $10-11 / 100,000$ population per year in western populations ${ }^{[2]}$. IAs rupture and subsequent hemorrhage may account for a mortality rate of $35 \%$, and most survivors are left with considerable neurological impairment ${ }^{[3-4]}$. Unruptured IAs are commonly treated through endovascular intervention or neurosurgical procedures to decrease the possibility of subarachnoid hemorrhage. However, most unruptured IAs are asymptomatic, and patients ignore potential risks. Thus, IAs must be diagnosed accurately before rupture, and the pathological mechanism of aneurysm formation should be emphasized.

Periodontitis and gingivitis are chronic inflammatory condition leading to the irreversible destruction of the tooth supporting tissues (gingiva, periodontal ligaments, and alveolar bone) with tooth loss as a common end point ${ }^{[5-7]}$. Periodontitis results from the complex pathological reactivity between chronic bacterial infection and host inflammatory response ${ }^{[8]}$. Gingivitis is defined as the inflammatory injury in the gingiva that are most commonly induced by the accumulation of dental plaque. In recent years, references have investigated the correlation between systematic vascular diseases and periodontitis or 
gingivitis. Lockhart et al. reported that oral bacterial deoxyribonucleic acid (DNA) had been extracted in peripheral arteries, and suggested that oral infections might contribute to vascular wall inflammation ${ }^{[9]}$. Bacteria from periodontal pockets and their secreta, such as endotoxins, have been identified in cardiovascular atherosclerotic lesions ${ }^{[10-12]}$. Iwai investigated periodontal bacterial DNA in abdominal aortic aneurysmal walls and then concluded that poor periodontal conditions have important effects on the progression of abdominal aortic aneurysms ${ }^{[13]}$.

Additional nine studies confirmed the significant correlation between periodontal diseases and cerebravascular strokes ${ }^{[14-22]}$. Further clinical studies speculated that periodontal inflammation caused by streptococcus mutans participates in the occurrence of intracranial hemorrhage strokes ${ }^{[23-25]}$. However, evidence for the role of periodontal diseases in IAs was insufficient because of the small sample sizes. Therefore, we performed this case-control study to investigate whether periodontal diseases were associated with IA formation and tried to discover novel epidemiological evidence.

\section{Method}

Study population

From September 2015 to September 2018, we collected cases that were admitted to our institution for IAs diagnosis via digital subtraction angiography(DSA) or computer tomography angiography(CTA) examination. During the same period, we also recruited cases that were diagnosed without IAs or clinical trial volunteers. A total of 281 cases signed written informed consent and were divided into two groups (166 cases in the IAs group and 115 cases in the non-IAs group). The privacy of the patients was strictly protected, and the protocol of this study was approved by our ethics committee. Periodontal health examination were provided to all recruited cases through a standardized approach in a dental unit by using a standard dental light, compressed air, a mouth mirror, and digital panoramic radiography free of charge. Periodontal disease was diagnosed on the basis of the clinical and radiographic criteria described by the 1999 consensus classification of periodontal diseases ${ }^{[26]}$.

Inclusion criteria were as follows: (1) individuals who were 18-80 years of age, (2) individuals with IAs and hospitalized volunteers (non-IAs) who wanted to participate in this study, and (3) individuals who underwent the examination of periodontal health condition. Exclusion criteria included (1) individuals with acute ruptured aneurysms; (2) individuals receiving antihypertensive therapy with calcium channel blockers, such as nifedipin; (3) individuals with severe cardiovascular diseases or cerebral ischemic stroke; (4) individuals with malignant diseases, chronic inflammatory diseases, or antibiotic use within 2 weeks; and (5) individuals missing clinical follow-up. Baseline demographic information (including age, sex, clinical presentation, cerebrovascular history, smoking and drinking history, diabetes, hypertension, and hypercholesterolemia) and IAs characteristics (location, size, shape, and quantity) were recorded.

Assessment of periodontal diseases 
Periodontal diseases mainly included periodontitis and gingivitis. Gingivitis severity was assessed by using the gingival index $(\mathrm{Gl})$ system based on the various tendencies of gingival bleeding after gingival irritation. A high index number (>1.1) was defined as severe gingivitis. Periodontitis was evaluated by using the parameters plaque index (PI), clinical attachment loss (CAL), and alveolar bone loss ( $\mathrm{ABL}$ ). Plaque and gingivitis were scored at four sites per tooth (buccal, mesiolingual, lingual, and distolingual) and averaged for each subject. We presented these parameters in accordance with the following definitions: first, the CAL was examined by inserting the tip of a community periodontal index (CPI) probe to measure the distance between the pocket and cementoenamel junction. Attachment levels were analyzed as continuous variables, and mean CAL $>4 \mathrm{~mm}$ was considered as severe periodontitis. Second, dental plaque was scored in accordance with the PI system, which is based on the same principle as the GI system. We divided PI into 4 sites $(\leq 0.5,0.51-1.0,1.01-1.5$, and $>1.5)$. A high score represented severe periodontitis. Third, $A B L$ levels were measured as the distance from the cementoenamel junction to the most apical extension of the bony defect. We stratified the $A B L$ into $<3.00$, $3.00-4.00,4.00-5.00$, and $>5.00 \mathrm{~mm}$.

Statistical analysis

Data were presented as mean \pm standard deviation or expressed in terms of frequencies and percentages. Independent sample t-test and chi-square test were performed for differences in continuous or categorical variables between the IAs group and the non-IAs group, respectively. Subgroup univariate and multivariate logistic regression analyses were performed to identify the independent contribution of gingivitis and periodontitis parameters (including $\mathrm{Gl}, \mathrm{CAL}, \mathrm{PI}$, and $\mathrm{ABL}$ ) to the incidence of IAs. Odds ratios (ORs) and 95\% confidence intervals (Cls) were given for all periodontal parameters. A two-sided $p$ value of $<0.05$ was considered to be significant. Statistical analysis was performed by using SPSS 22.0 software (SPSS Inc., Chicago, IL, USA).

\section{Results}

A total of 281 cases (including 174 [61.9\%] females and 107 [38.1\%] males) were recruited in this study. The age ranged from 18 years to 79 years (51.34 \pm 10.56 years). Table 1 showed the baseline characteristics and subgroup analysis results of the IAs group and non-IAs group. There were no statistically significant differences in the distributions of sex, diabetes mellitus, hyperlipidemia, cerebrovascular history (including cerebral ischemic and hemorrhage events), other personal history (such as alcohol, smoking, and BMI) in the two subgroups (all $p>0.05$ ). Chi-square tests indicated that cases in the IAs group were older than those in the non-IAs group ( $53.95 \pm 8.56$ vs $47.79 \pm 12.33, p<$ 0.001 ) and had a higher incidence of hypertension (76 vs $34, p=0.006$ ).

Univariate regression analysis revealed that aged cases (over 50 years) combining with these periodontal parameters and hypertension, were significantly associated with the occurrence of IAs (all $p<0.05$ ) (Table 2). The parameters of periodontal diseases ( $\mathrm{GI}, \mathrm{CAL}, \mathrm{PI}$, and $\mathrm{ABL}$ ) were divided into four layers on the basis of their averages and referenced literature ${ }^{[27]}$. Table 3 demonstrated that severe periodontitis 
and gingivitis might be accompanied by the higher risk of IAs formation. GI could be considered as a risk predictive factor of IAs (OR of GI >1.1 17.11,95\% Cl 3.339-87.66, p=0.001). Higher PI (mainly > 1.5) showed the similar correalation (OR 6.968,95\% Cl 2.396-20.259, p囚0.001). High CAL (>4.00 mm), which indicated periodontitis with increased severity, was accompanied by the high risk of IA (OR $4.074,95 \% \mathrm{Cl}$ $1.012-16.391, p=0.048)$. The severity of $A B L(>4.00 \mathrm{~mm})$ represented another significant risk factor of IAs with two OR values, specifically, 4.00-5.00 mm ( $p=0.003)$, which corresponded to an OR of 6.409 , and over $5.0 \mathrm{~mm}(p=0.005)$, which corresponded to an OR of 21.835 . The values of $95 \% \mathrm{Cl}$ are shown in Table 3. All of the above parameters of severe periodontal diseases had $p<0.05$. While comparing with

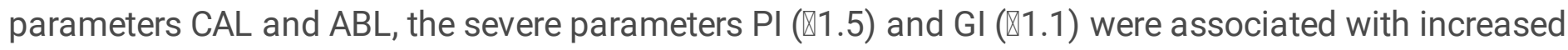
diagnostic value of aneurysms formation according to multivariate logistic regression analyses (Table 4, $\mathrm{p} \otimes 0.001$ and $\mathrm{p} \otimes 0.007)$.

\section{Discussion}

Periodontitis and gingivitis are associated with the risk of several diseases, such as rheumatoid arthritis and atherosclerosis ${ }^{[28-29]}$. Recent studies have discussed the role of periodontal diseases in causing cerebral ischemia. Chiu demonstrated that periodontitis was associated with stroke which commonly caused by large-artery atherosclerosis; this result supported the hypothesis of a possible link between periodontitis and atherosclerosis ${ }^{[30]}$. After adjusting for confounding vascular factors on the basis of etiologic subgroup analysis, Grau et al. concluded that severer periodontitis was an independent risk predictive factor for IAs with atherothrombotic origins (OR 2.35 [1.00-11.0] and OR 13.2 [2.68-64.7]) and that gingivitis was independently associated with cerebral ischemia given its value as an indicator of the actual status of periodontal inflammation ${ }^{[31]}$. These findings suggested that chronic periodontal inflammatory responses contributed to a prothrombotic state via recurrent bacteremia and platelet or endothelial activation. Plaque destabilization is a potential trigger of cardioembolism and cryptogenic stroke ${ }^{[32-33]}$. In addition, systemic immunization induced by local periodontitis may influence the accumulation of oxidized lipids in vascular walls and subsequent atherosclerotic remodeling indirectly.

Periodontal diseases, especially periodontitis, are associated with the remodeling of the aneurysmal wall in abdominal aortic aneurysms. In experimental models, periodontal bacteria promote the degeneration of the abdominal aortic aneurysmal wall by increasing the recruitment of neutrophils to the intraluminal thrombus that covers the inner portion of the abdominal aortic aneurysm ${ }^{[34]}$. The pathology of IA and abdominal aortic aneurysm shares several features ${ }^{[35]}$. The current understanding is that IAs formation as the end result of flow-driven inflammatory cell-mediated cerebral artery wall remodeling at sites where high flow exerts high wall shear stress ${ }^{[1]}$. However, IA is not detected in all cases under high flow and shear stress in the bifurcations of cerebral arteries. Previous studies on the correlation between periodontal diseases and stroke focused on the pathological mechanism of IA. Pyysalo detected the presence of oral bacteria DNA in ruptured and unruptured intracranial aneurysmal walls firstly ${ }^{[36]}$. Hallikainen et al. speculated that periodontitis predisposes the artery wall toward aneurysm development ${ }^{[37]}$. However, few works have paid attention to the relationship between intracranial 
hemorrhagic diseases and periodontal diseases. In this case-control study, we investigated the potential correlation between periodontal diseases (including periodontitis and gingivitis) and IAs formation. Although $\mathrm{GI}, \mathrm{PI}, \mathrm{CAL}$, and $\mathrm{ABL}$ have long been known as actual indicators of periodontal inflammation caused by multitudinous oral bacteria, we considered that these parameters were independent predictive risk factors of IAs formation firstly. Further subgroup analysis demonstrated that severe periodontitis parameters might result in the high incidence of IAs. A past observation discussed that systemic elastase activity might play an important role given that increased serum elastase concentrations were associated with IAs, although the source of serum elastase was unknown ${ }^{[38]}$. Potential sources for circulating elastase were macrophages or neutrophils ${ }^{[39]}$. Tooth brushing or chewing could disseminate periodontal porphyromonas gingivalis (PG), especially in patients with periodontitis or gingivitis, to extraoral sites via circulation and then induce systemic inflammatory responses through transient bacteremia ${ }^{[40]}$. PG is available for modifying dendritic cell function and cause proinflammatory cytokine production in macrophages. Thus, periodontal pathogens that infiltrating cerebral arteries likely lead to excessive collagen degradation and neutrophil accumulation in the thrombus, promoting changes in the course of cerebral artery remodeling ${ }^{[41]}$. In another mechanism, neutrophils induced by periodontal pathogens are a major source of proteolytic activity because they release the matrix metalloproteinases 8 (MMP-8) and MMP-9, myeloperoxidase, and elastase and then accelerate the course of proteolytic or cytotoxic injury ${ }^{[42]}$. The ultimate pathology is damage to the elastic fibers of smooth muscles and the degeneration or necrosis of the intima medium. In combination with chronic hypertension, external vascular walls swell to form IAs.

Previous literature has indicated that aged cases (especially over 50 years) and hypertension are risk predictive factors of IAs formation ${ }^{[43-44]}$. In the present study, we included patients who were older than 50 years or suffered hypertension and found that these cases had an increased incidence of IAs. Systemic hypertension not only affects tissue remodeling or vascular wall inflammation by exerting abnormal hemodynamic stresses but also activates the local renin-angiotensin system ${ }^{[45]}$. Moreover, hypertension may mediate vascular inflammation through the activation of NF-kappa B, which can further promote inflammation ${ }^{[46]}$. The continuous stimulatory response caused by hypertension and inflammation can result in the degeneration of vascular walls and subsequent IA formation.

This study has several limitations. First, although the literature has indicated that periodontal treatment might induce bacteria to transfer to systematic circulation, we could not persuade all patients to undergo periodontal or caries treatment because of their poor obedience. Thus, we will further study whether periodontal treatment has low relevance to IA. Second, the specific values of periodontal disease parameters that have the optimal predictive values for the incidence of IAs should be quantified through receiver operating characteristic curve analysis. Third, we did not perform further experimental research on inflammatory mediators that might cause IA directly. Fourth, other studies have demonstrated that age and hypertension were associated with the high risk of periodontal diseases. In this study, we only attempted to investigate the mechanism of aneurysm formation and neglected the relationship between these two factors and periodontal diseases. 


\section{Conclusion}

Periodontal diseases are significantly associated with the high incidence of IA. Given our results, patients who suffer from severe gingivitis or periodontitis and hypertension should be encouraged to undergo cerebrovascular examination. Mechanistic experiments on inflammatory response will positively predict the risk of IAs formation.

\section{List Of Abbreviations}

ABL: alveolar bone loss

BMl: body mass index

CAL: clinical attachment loss

Cls: confidence intervals

CPI: community periodontal index

CTA: computer tomography angiography

DNA: deoxyribonucleic acid

DSA: digital subtraction angiography

Gl: gingival index

IA: intracranial aneurysm

MMP: matrix metalloproteinases

OR: Odds ratios

PI: plaque index

PG: porphyromonas gingivalis

SAH: subarachnoid hemorrhage

\section{Declarations}

Ethics approval: Institutional review board approval was obtained from Beijing Tiantan Hospital's ethics committee for this study.

Consent for publication: Not applicable. 
Availability of data and materials: The datasets used and/or analysed during the current study are available from the corresponding author on reasonable request.

Competing interests: The authors declare that they have no competing interests.

Funding: This study was supported by the National Natural Science Foundation (81471166) and Capital Medical Development Research Foundation of China (2016-1-1075).

Contributorship Statement: Author contributions to the study and manuscript preparation include the following.

Youxiang Li and Huijian Ge conceived the study design.

Keyun Liu and Jia Sun completed the collection and collation of the original data.

Keyun Liu, Jia Sun, Huijian Ge filtered the final data according to the criteria.

Keyun Liu and Lingling Shao analyzed and sorted out the data.

Keyun Liu and Huijian Ge drafted and wrote the manuscript.

Youxiang Li and Huijian Ge supervised the project, obtained the research grants, made key revisions and final approvals to the manuscript.

All authors made substantial contributions to the manuscript, read and approved the final version of manuscript.

Acknowledgements: We are grateful to Dr. Aihua Liu, Dr. Peng Jiang, Dr. Shiqing Mu, Dr. Ming Lv and Dr. Jingbo Zhang for the endovascular treatment and data.

\section{References}

1. Joona Hallikainen, Sara Keränen, Jarno Savolainen, Matti Närhi, Anna Liisa Suominen, Pekka Ylöstalo, et al. Role of oral pathogens in the pathogenesis of intracranial aneurysm: review of existing evidence and potential Neurosurg Rev.2020;10.1007/s10143-020-01253-y

2. Ingall $\mathrm{T}$, Asplund $\mathrm{K}$, Mahonen $\mathrm{M}$, Bonita R. A multinational comparison of subarachnoid hemorrhage epidemiology in the WHO MONICA stroke study. Stroke. 2000;31:1054-1061.

3. Brisman JL, Song JK, Newell DW. Cerebral aneurysms. N Engl J Med. 2006;355(9): 928-939.

4. Vlak MH, Algra A, Brandenburg R, Rinkel G. Prevalence of unruptured intracranial aneurysms, with emphasis on sex, age, comorbidity, country, and time period: a systematic review and meta-analysis. Lancet Neurol. 2011;10(7):626-636.

5. de Molon RS, Rossa C Jr, Thurlings RM, Cirelli JA, Koenders MI. Linkage of periodontitis and rheumatoid arthritis: current evidence and potential biological interactions. Int J Mol 
Sci.2019;20(18):pii: E4541.

6. Kinane DF, Stathopoulou PG, Papapanou PN. Periodontal diseases. Nat. Rev. Dis. Primers. 2017;3:17038.

7. Correa JD, Fernandes GR, Calderaro DC, Mendonca SM, Silva JM, Albiero ML, et al. Oral microbial dysbiosis linked to worsened periodontal condition in rheumatoid arthritis patients. Sci Rep. 2019;9:8379.

8. Kinane DF. Causation and pathogenesis of periodontal disease. Periodontol.2001;25:8-20.

9. Lockhart PB, Brennan MT, Sasser HC, Fox PC, Paster BJ, Bahrani-Mougeot FK. Bacteremia associated with toothbrushing and dental extraction. Circulation. 2008;117(24): 3118-3125.

10. Wilson W, Taubert KA, Gewitz M, Lockhart PB, Baddour LM, Levison M, et al. Prevention of infective endocarditis: guidelines from the American Heart Association: a guideline from the American Heart Association Rheumatic Fever, Endocarditis, and Kawasaki Disease Committee, Council on Cardiovascular Disease in the Young, and the Council on Clinical Cardiology, Council on Cardiovascular Surgery and Anesthesia, and the Quality of Care and Outcomes Research Interdisciplinary Working Group. Circulation. 2007;116(15):1736-1754.

11. Hirschfeld J, Kawai T. Oral inflammation and bacteremia: implications for chronic and acute systemic diseases involving major organs. Cardiovasc Hematol Disord Drug Targets. 2015;15(1):7084.

12. Teles R, Wang CY. Mechanisms involved in the association between periodontal diseases and cardiovascular disease. Oral Dis. 2011;17(5):450-461.

13. Iwai T. Periodontal bacteremia and various vascular diseases. J Periodontal Res. 2009;44(6): 689694.

14. Hosaka K, Hoh BL. Inflammation and cerebral aneurysms. Transl Stroke Res. 2014;5(2):190-198.

15. Frosen J, Tulamo R, Paetau A, Laaksamo E, Korja M, Laakso A, et al. Saccular intracranial aneurysm: pathology and mechanisms. Acta Neuropathol 2012;123(6):773-786.

16. Kanematsu Y, Kanematsu M, Kurihara C, Tada Y, Tsou TL, van Rooijen N, et al. Critical roles of macrophages in the formation of intracranial aneurysm. Stroke. 2011;42(1):173-178.

17. Aoki T, Kataoka H, Morimoto M, Nozaki K, Hashimoto Macrophage-derived matrix metalloproteinase2 and -9 promote the progression of cerebral aneurysms in rats. Stroke. 2007;38(1):162-169.

18. Gounis MJ, van der Marel K, Marosfoi M, Mazzanti ML, Clarençon F, Chueh JY, et al. Imaging Inflammation in Cerebrovascular Disease. Stroke. 2015;46(10):2991-2997.

19. Schenkein HA, Loos BG. Inflammatory mechanisms linking periodontal diseases to cardiovascular diseases. J Clin Periodontol. 2013;40 Suppl 14: S51-69.

20. Pihlstrom BL, Michalowicz BS, Johnson NW. Periodontal diseases. Lancet. 2005;366(9499):18091820.

21. Kinane DF. Causation and pathogenesis of periodontal disease. Periodontol. 2000;2001,25:8-20. 
22. Noack B, Genco RJ, Trevisan M, Grossi S, Zambon JJ, De Nardin E. Periodontal infections contribute to elevated systemic C-reactive protein level. J Periodontol. 2001;72(9):1221-1227.

23. Nakano K, Hokamura K, Taniguchi N, Wada K, Kudo C, Nomura R, et al. The collagen-binding protein of Streptococcus mutans is involved in haemorrhagic stroke. Nat Commun. 2011;2: 485.

24. Tonomura S, Ihara M, Kawano T, Tanaka T, Okuno Y, Saito S, et al. Intracerebral hemorrhage and deep microbleeds associated with cnm-positive Streptococcus mutans; a hospital cohort study. Sci Rep. 2016;6:20074.

25. Kim HD, Sim SJ, Moon JY, Hong YC, Han DH. Association between periodontitis and hemorrhagic stroke among Koreans: a case-control study. J Periodontol. 2010;81(5):658-665.

26. Armitage GC. Development of a classification system for periodontal diseases and conditions. Ann Periodontal. 1999;4:1-6.

27. Fontaine V, Jacob MP, Houard X, Rossignol P, Plissonnier D, Angles-Cano E, et al. Involvement of the mural thrombus as a site of protease release and activation in human aortic aneurysms. Am J Pathol. 2002;161(5):1701-1710.

28. Karin Lundberg1, Natalia Wegner, Tülay Yucel-Lindberg, Patrick J Venables. Periodontitis in RA-the citrullinated enolase connection. Nat Rev Rheumatol. 2010;6(12):727-30.

29. M Kebschull, R T Demmer, P N Papapanou. “Gum bug, leave my heart alone!"-epidemiologic and mechanistic evidence linking periodontal infections and atherosclerosis. J Dent Res. 2010;89(9):879902.

30. Chiu B. Multiple infections in carotid atherosclerotic plaques. Am Heart J. 1999;138:S534-S536.

31. Grau AJ, Becher H, Ziegler CM, Lichy C, Buggle F, Kaiser C, et al. Periodontal Disease as a Risk Factor for Ischemic Stroke. Stroke. 2004;35:496-501.

32. Leira Y, Seoane J, Blanco M, Rodríguez-Yáñez M, Takkouche B, Blanco J, et al. Association between periodontitis and ischemic stroke: a systematic review and meta-analysis. Eur $\mathrm{J}$ Epidemiol. 2017;32(1):43-53.

33. Lourbakos A, Yuan YP, Jenkins AL, Travis J, Andrade-Gordon P, Santulli R, et al. Activation of protease-activated receptors by gingipains from Porphyromonas gingivalis leads to platelet aggregation: a new trait in microbial pathogenicity. Blood. 2001;97:3790-3797.

34. Delbosc S, Alsac JM, Journe C, Louedec L, Castier Y, Bonnaure-Mallet M, et al. Porphyromonas gingivalis participates in pathogenesis of human abdominal aortic aneurysm by neutrophil activation. Proof of concept in rats. PLoS One. 2011;13(6):e18679.

35. Marbacher S, Marjamaa J, Bradacova K, von Gunten M, Honkanen P, Abo-Ramadan U, et al. Loss of mural cells leads to wall degeneration, aneurysm growth, and eventual rupture in a rat aneurysm model. Stroke. 2014;45(1):248-254.

36. Pyysalo MJ, Pyysalo LM, Pessi T, Karhunen PJ, Öhman The connection between ruptured cerebral aneurysms and odontogenic bacteria. J Neurol Neurosurg Psychiatry. 2013;84(11):1214-1218. 
37. Hallikainen J, Lindgren A, Savolainen J, Selander T, Jula A, NärhiM, et al. Periodontitis and gingival bleeding associate with intracranial aneurysms and risk of aneurysmal subarachnoid hemorrhage. Neurosurg Rev. 2019 Apr 10. doi: 10.1007/s10143-019-01097-1.

38. Connolly ES Jr, Fiore AJ, Winfree CJ, Prestigiacoma CJ, Goldman JE, Solomon RA. Elastin degradation in the superficial temporal arteries of patients with intracranial aneurysms reflects changes in plasma elastase. Neurosurgery. 1997;40(5):903-8.

39. Delbosc S, Alsac JM, Journe C, Louedec L, Castier Y, Bonnaure-Mallet M, et al. Porphyromonas gingivalis participates in pathogenesis of human abdominal aortic aneurysm by neutrophil activation. PLoS One. 2011;6(4):e18679.

40. Forner $L$, Larsen $T$, Kilian $M$, Holmstrup Incidence of bacteremia after chewing, tooth brushing and scaling in individuals with periodontal inflammation. J Clin Periodontol. 2006;33(6):401-407.

41. Shimada K, Furukawa H, Wada K, Korai M, Wei Y, Tada Y, et al. Protective role of peroxisome proliferator-activated receptor-үin the development of intracranial aneurysm rupture. Stroke. 2015;46(6):1664-1672.

42. Vincent Fontaine, Marie-Paule Jacob, Xavier Houard, Patrick Rossignol, Didier Plissonnier, Eduardo Angles-Cano, et al. Involvement of the mural thrombus as a site of protease release and activation in human aortic aneurysms. Am J Pathol. 2002;161(5):1701-10.

43. Juvela S. Prevalence of and risk factors for intracranial aneurysms. Lancet Neurol. 2011;10(7):595597.

44. Schievink WI. Intracranial aneurysms. N Engl J Med. 1997;336(1): 28-40.

45. Tada Y, Wada K, Shimada K, Makino H, Liang El, Murakami S, et al. Roles of hypertension in the rupture of intracranial aneurysms. 2014;45(2):579-586.

46. Aoki T, Nishimura M, Kataoka H, Ishibashi R, Nozaki K, Hashimoto Reactive oxygen species modulate growth of cerebral aneurysms: A study using the free radical scavenger edaravone and p47phox $(-/-)$ mice. Lab Invest. 2009;89:730-741.

\section{Tables}

Table 1. Baseline Characteristics of Patients 
1. Value

$$
\mathrm{IA}(+) \text { (IA+ vs IA-) }
$$

Variable Total, $\mathrm{n}=281 \quad \mathrm{n}=166 \quad \mathrm{IA}(-), \mathrm{n}=115$

\begin{tabular}{|c|c|c|c|c|}
\hline Mean age (years) & $\begin{array}{c}51.34 \pm \\
10.56\end{array}$ & $\begin{array}{c}53.95 \pm \\
8.56\end{array}$ & $\begin{array}{c}47.79 \pm \\
12.33\end{array}$ & $<0.001$ \\
\hline Female sex, n (\%) & $174(61.9)$ & $99(59.6)$ & $75(65.2)$ & 0.383 \\
\hline Hypertension, n (\%) & $110(39.1)$ & $76(45.8)$ & $34(29.6)$ & 0.006 \\
\hline Diabetes mellitus, n (\%) & $26(9.3)$ & $11(6.6)$ & $15(13.0)$ & 0.093 \\
\hline Hyperlipidemia, n (\%) & $26(9.3)$ & $17(10.2)$ & $9(7.8)$ & 0.537 \\
\hline Cerebrovascular diseases history, n (\%) & $18(6.4)$ & $10(6.0)$ & $8(6.9)$ & 0.807 \\
\hline Heavy smoking history, n (\%) & $54(19.2)$ & $36(21.7)$ & $18(15.7)$ & 0.222 \\
\hline Bibulosity, n (\%) & $39(13.9)$ & $19(11.4)$ & $20(17.4)$ & 0.165 \\
\hline $\begin{array}{l}\text { Family history of cerebrovascular diseases, } \mathrm{n} \\
(\%)\end{array}$ & $30(10.7)$ & $21(12.7)$ & $9(7.8)$ & 0.241 \\
\hline Mean BMI & $24.89 \pm 4.06$ & $25.18 \pm 4.25$ & $24.46 \pm 3.77$ & 0.145 \\
\hline
\end{tabular}

Data are shown as mean \pm SD or absolute and Chi-square test between 2 groups;

BMI: Body Mass Index;

Cerebrovascular diseases history was defined as the patients who presented with cerebral ischemic and hemorrhage before inpatient

Table 2. Univariate logistic regression analysis of clinical characteristics and periodontitis parameters for intracranial aneurysm formation

\begin{tabular}{ccccc}
\hline & \multicolumn{2}{c}{ univariate regression analysis } \\
Variables & P-value & OR & \multicolumn{2}{c}{$95 \%$ CI } \\
\hline Age(口 50 years) & $<0.001$ & 1.047 & 1.022 & 1.073 \\
Hypertension & 0.006 & 2.047 & 1.232 & 3.401 \\
\hline GI & $<0.001$ & 11.428 & 5.318 & 24.557 \\
\hline PI & $<0.001$ & 3.053 & 1.908 & 4.885 \\
CAL & 0.001 & 1.794 & 1.252 & 2.571 \\
\hline ABL & $<0.001$ & 2.211 & 1.613 & 3.030 \\
\hline
\end{tabular}

OR: Odds Ratio; 95\% CI: 95\% Confidence Interval; 
GI: gingival index; PI: plaque index; CAL: clinical attachment loss;

ABL: alveolar bone loss;

Table 3. Univariate logistic regression analysis for the correlation between four layers of periodontitis parameters and intracranial aneurysm

\begin{tabular}{|c|c|c|c|c|c|}
\hline & Variable & OR & $95 \%$ & ${ }_{6} \mathrm{CI}$ & P-value \\
\hline & & & Lower limit & Upper limit & \\
\hline & $<0.3^{a}$ & & & & \\
\hline & $0.3-0.7$ & 0.553 & 0.258 & 1.185 & 0.128 \\
\hline & $0.71-1.1$ & 2.034 & 0.844 & 4.905 & 0.114 \\
\hline GI & $>1.1$ & 17.110 & 3.339 & 87.660 & 0.001 \\
\hline & $\leq 0.5^{b}$ & & & & \\
\hline & $0.51-1.0$ & 0.687 & 0.341 & 1.384 & 0.294 \\
\hline & $1.01-1.5$ & 0.852 & 0.349 & 2.084 & 0.726 \\
\hline PI & $>1.5$ & 6.968 & 2.396 & 20.259 & $<0.001$ \\
\hline & $<3 \mathrm{~mm}^{\mathrm{c}}$ & & & & \\
\hline & $3.00-3.50 \mathrm{~mm}$ & 1.001 & 0.509 & 1.970 & 0.997 \\
\hline & $3.50-4.00 \mathrm{~mm}$ & 0.433 & 0.176 & 1.061 & 0.067 \\
\hline CAL & $>4.00 \mathrm{~mm}$ & 4.074 & 1.012 & 16.391 & 0.048 \\
\hline & $<3 \mathrm{~mm}^{\mathrm{d}}$ & & & & \\
\hline & $3.00-4.00 \mathrm{~mm}$ & 1.410 & 0.775 & 2.564 & 0.260 \\
\hline & $4.00-5.00 \mathrm{~mm}$ & 6.409 & 1.907 & 21.533 & 0.003 \\
\hline ABL & $>5.00 \mathrm{~mm}$ & 21.835 & 2.503 & 190.463 & 0.005 \\
\hline
\end{tabular}

Adjusted with age, hypertension. OR: Odds Ratio; 95\% CI: 95\% Confidence Interval;

a " $<0.3$ " used as reference group in the binary logistic regression.

b " $\leq 0.5$ " used as reference group in the binary logistic regression.

c " $<3 \mathrm{~mm}$ " used as reference group in the binary logistic regression.

$\mathrm{d}$ " $<3 \mathrm{~mm}$ " used as reference group in the binary logistic regression.

GI: gingival index; PI: plaque index; CAL: clinical attachment loss;

ABL: alveolar bone loss; 
Table 4 Multiple logistic regression analysis for the correlation between severe periodontitis parameters and intracranial aneurysm

\begin{tabular}{cclll}
\hline Variable & OR & \multicolumn{2}{c}{$95 \%$ CI } & P-value \\
& & Lower limit & Upper limit & \\
\hline GI $>1.1$ & 8.001 & 1.751 & 36.557 & 0.007 \\
PI $>1.5$ & 6.438 & 2.381 & 17.406 & $<0.001$ \\
\hline $\mathrm{CAL}>4.00 \mathrm{~mm}$ & 2.360 & 0.600 & 9.287 & 0.219 \\
\hline $\mathrm{ABL}>4.00 \mathrm{~mm}$ & 7.989 & 0.958 & 66.600 & 0.055 \\
\hline
\end{tabular}

OR: Odds Ratio; 95\% CI: 95\% Confidence Interval;

GI: gingival index; PI: plaque index; CAL: clinical attachment loss;

ABL: alveolar bone loss; 